\title{
Design and Characterization of Single-Mode Microstructured Fibers with Improved Bend Performance
}

\author{
Vladimir Demidov, Konstantin Dukel'skii and Victor Shevandin \\ S.I. Vavilov Federal Optical Institute, St. Petersburg \\ Russia
}

\section{Introduction}

Over the last few years, clear progress has been made in research and development of single-mode optical fibers with a large core (when core diameter exceeds $10 \mu \mathrm{m}$ ). Such advances were stimulated essentially by growing requirements for means of high power laser radiation transmission. The urgent problem of laser beam delivery lies in the necessity of the primary Gaussian power distribution of light inherent to many laser sources to be maintained without both temporal and spatial distortions. So optical fibers that support only a single transverse mode prove to be the most appropriate technique for efficient light transfer in production areas of complex or compact architecture. But there are still a number of limitations to cope with. For instance, as the power density of generated laser beams increases, the fiber core has to be expanded adequately in order to minimize the impact of undesirable nonlinear effects such as Raman scattering, Brillouin scattering and self-phase modulation. Moreover, fiber material will exhibit irreversible breakdown if the power level equals or exceeds the critical damage threshold.

Conventional single-mode fibers with step-index or graded-index refractive index profile can be acceptably adapted for the realization of large cores. However, the core dimensions enlargement permanently results in the reduction of the refractive index difference between the core and the cladding $(\Delta \mathrm{n})$. This, in turn, affects adversely the numerical aperture of the fiber (NA), which then has to be reduced twice from its standard values of larger than 0.1 to achieve core diameters of approximately $15 \mu \mathrm{m}$ at a wavelength around $1 \mu \mathrm{m}$ (Tunnermann et al., 2005). Such NA lowering weakens considerably the fiber waveguiding so the optical fiber becomes very sensitive to various perturbations, especially to bending effects. Further decrease of NA will require keeping the uniformity of the core refractive index in the vicinity of $10^{-4}-10^{-5}$. It is technologically unattainable when using chemical vapor-phase deposition methods for the fiber preform fabrication.

An alternative flexible approach to solve this challenge is based on exploiting unique wave guiding properties of microstructured optical fibers (MOFs), also known as photonic crystal fibers or holey fibers. MOF design can relatively easily provide extended cores and hence large effective mode areas that nowadays reach values of even thousands of $\mu \mathrm{m}^{2}$. This phenomenon perfectly coordinates with the ability to manage accurately the effective $\Delta \mathrm{n}$ value at a level of as low as 0.0001 or less. Furthermore, MOFs, as opposed to single-mode 
fibers of a conventional design, can assure robust fundamental mode propagation over a broad wavelength range within the transparency window of silica. The only restriction has to be taken into account while manufacturing microstructures with a large core relates to reasonable control over spectral position of bend-loss edge crucial for the fiber application potential.

Typical silica-based MOF structure is defined by a certain number of air holes arranged in a regular triangular lattice running along the entire length of the fiber (Knight et al., 1996, 1997). One missing central hole filled with a glass introduces a defect in the lattice, acting as the guiding core with the refractive index of undoped fused silica. Holes surrounding the core area serve as the cladding with the effective refractive index lower than that of the core due to presence of the air. Provided that the degree of air content expressed commonly by the k-parameter (i.e. the ratio of the air hole diameter $d$ to the lattice pitch $\Lambda$ ) does not exceed 0.45 (Mortensen, 2002), the fiber supports a single transverse mode for any wavelength (the endlessly single mode regime). The most suitable manner of core expansion is scaling of cross-sectional fiber dimensions without changes in the given lattice structure. But on the understanding that the fundamental mode acts as the leaky one due to bending in the short-wavelength region, the position of bend-induced leakage boundary moves to longer wavelengths while increasing the core size (Nielsen et al., 2004b). Consequently, the spectral operation range steadily narrows that provokes fibers to be allocated on spools of greater radiuses. This prevents MOFs from being widely exploited in industrial laser or beam delivery applications with standardized curve parameters.

In this work we have concentrated our efforts on finding and implementation of a few novel MOF designs that could effectively combine the large core dimensions and the expanded spectral operation range as compared to classical MOFs. It is obvious that new structures should be actualized by applying principles different from the basic concepts of the standard MOF technology. Here we will focus on two special approaches: 1) competent manipulation of $\Lambda$-parameter in the selected wavelength region; 2 ) ensuring proper fiber conditions for the establishment of a substantial difference in attenuation coefficients of the fundamental $\left(\mathrm{LP}_{01}\right)$ and the higher order ( $\left.\mathrm{LP}_{11}\right)$ modes (differential modal attenuation).

\section{MOFs with a multi-element core}

\subsection{Background on large-core structure design}

MOF guides light along the core via the modified total internal reflection mechanism similar to that of conventional single-mode fiber (Knight, 2003). However, in some cases MOF can demonstrate specialty features that have no analogies in conventional waveguide theory.

Our previous investigations (Dukel'skii et al., 2005, 2006) indicate that the lattice pitch $\Lambda$ (in general, the ratio of the wavelength $\lambda$ to the pitch $\Lambda$ ) is the most significant parameter responsible for such main optical property as the capability of light confinement. For MOFs with air holes assembled in a triangular pattern we revealed the discrete transition between the availability of wave guidance and the lack of it. The exact position of this transition strongly depended on the $\Lambda$ value.

Mentioned effect appeared in three forms: 1) absence of light canalization in short-length samples (Dukel'skii et al., 2005); 2) appreciable increase in attenuation coefficient of multimode samples with $\mathrm{k} \geq 0.8$ in the short-wavelength region of the spectra (Dukel'skii et al., 2006); 3) intensive short-wavelength leakage of the fundamental mode power into the outer fiber cladding in single-mode samples (Nielsen et al., 2004b). 
For example, we observed straight fiber segments of a length from 2 to 5 centimeters in a microscope under white light launched in each sample at arbitrary angles. When $\Lambda$, defined as the hole-to-hole spacing in the first air-hole ring, exceeded $\sim 10 \mu \mathrm{m}$, samples were characterized by the weakening of waveguiding properties in the visible part of the spectra (Dukel'skii et al., 2005). To be more precise, the core ceased to canalize light and the distribution of light over the fiber cross-section became totally uniform. No changes in the launch fiber conditions could modify that uniformity. Ultimately, the samples with $\mathrm{k} \geq 0.8$ supported light propagation, whereas the samples with $\mathrm{k}<0.6$ diverged.

It is a well-known fact that conventional optical fiber made of glass materials with different refractive indexes (as well as corresponding fiber preform) can guide light irrespective of its transverse dimensions. The core diameter enlargement leads only to the increase in amount of excited modes, but not to the absolute lack of waveguiding properties. MOF technology does not imply light guidance of the initial capillary stack (preform) due to its particular structure dissimilar to the resultant MOF design. At the same time, the ability of the fiber drawn from the stack to guide light strongly depends on the transverse microstructure dimensions ( $\mathrm{d}$ and $\Lambda$ ).

The next interesting phenomenon intrinsic to MOFs with a triangular cladding structure we detected after the long fiber samples (about 100 meters in length) had been investigated concerning the optical loss measurements (Figure 1).

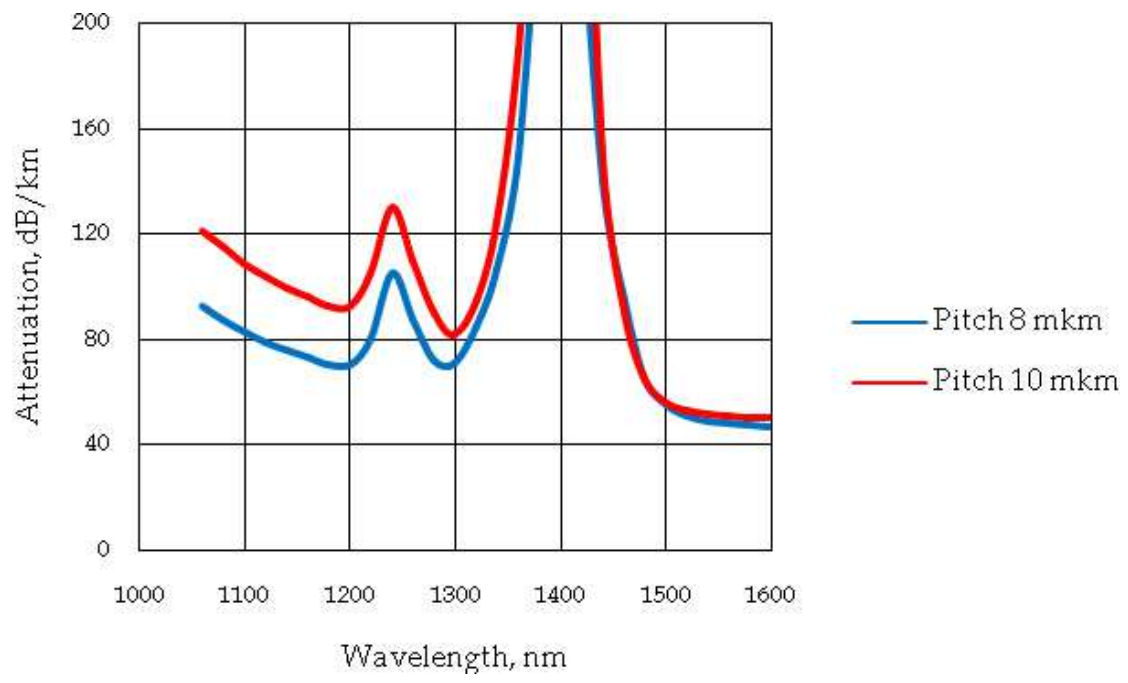

Fig. 1. Spectral attenuation pattern of the MOFs with $\mathrm{k} \geq 0.8$ depending on the $\Lambda$-parameter (Dukel'skii et al., 2006).

As Figure 1 shows, the increase in optical losses occurred according to the fiber diameter growth, especially in the short-wavelength part of the spectra. Evidently, the $\Lambda$-parameter increased in direct proportion to the outer fiber diameter expansion. So we had the same situation as described above: the increase in hole-to-hole spacing definitely impaired wave guidance. The effect was observed while handling only multimode fiber samples with $\mathrm{k} \geq$ 0.8 and was not connected with macrobending or microbending losses. 
The third aspect of $\Lambda$ manipulation included the fact that abovementioned short-wavelength leakage of the fundamental mode power can dramatically enhance when core diameter rises from 20 to $35 \mu \mathrm{m}$ (Nielsen et al., 2004b). In practical applications this unfavourable phenomenon forces MOFs to be placed on spools with augmented radiuses, extended, for example, from normal radius of 8 centimeters for communication fiber up to non-standard radius of 16 centimeters. The enhancement of optical power leakage can be formally interpreted by the impact of the $\Lambda$-parameter that proportionally increases with the fiber core diameter enlargement. So selecting suitable $\Lambda$ for a given spectral region, we could control the position of modal leakage boundary.

Thus, we found out that qualified adjustment of $\Lambda$-parameter in MOFs characterized by a large core size (up to $35 \mu \mathrm{m}$ ) can be promising for the purpose of deriving a set of special properties. However, aids and concepts for achievement and implementation of these features are not trivial. One of the feasible ways to improve light confinement is to comprise the core of several elements. In this case the core becomes bigger in comparison with the core of a typical MOF structure, although the outer fiber diameter remains permanent. On the basis of geometrical considerations, it is possible to substitute not one, as ordinary, but seven or nineteen central capillaries in the initial triangular array for one solid rod made of the same material as the fiber cladding. By means of the substitution method the values of $\Lambda$-parameter can be reduced by two (7 central capillaries) or three (19 central capillaries) times in the resultant MOF as compared to a standard 1-element-core analog of the same core size.

It should be noted that there are several publications (Limpert et al., 2005, 2006) reporting on development of 7- and 19-element-core MOFs for generation of high power laser radiation, but no detailed information about modal consistence or bending performance is provided. Superior theoretical analysis (Saitoh et al., 2005) shows that the endlessly single-mode regime of operation for the 7-element-core MOF can be realized under the higher order mode cut-off condition $\mathrm{k}<0.046$. Apparently, implementation and multiple reproduction of such tiny structure correspond with huge technological difficulties. Furthermore, it seems even more difficult to carry out practically the condition for the single-mode operation of the 19-element-core MOF, which is expected to be extremely low (continuing a row for the phase higher order mode cut-off condition for the 1-element-core structure $k<0.45$ and for the 7-element-core structure $\mathrm{k}<0.046$ ).

So taking into account the effect $\Lambda$-parameter has on the capability of light confinement, we stated a goal of creating a family of single-mode MOF structures with a large multi-element core that will not be subjected to strong influence of macrobending losses.

\subsection{MOFs with the core comprised of 7 missing holes}

In the first stage of our research we have successfully produced a series of MOFs with the core design presented in Figure 2. The arrangement included the 7-element core area and five air-hole rings organizing the light-reflecting cladding. All experimental samples were drawn from capillary stacks using commercially-available synthetic silica tubes and rods with $\mathrm{OH}$-content in concentration of several ppm. The required value of k-parameter was obtained by the appropriate adjustment of capillary pressure in the high temperature zone of the stack during the drawing process. The outer surfaces of the elements were purified beforehand in order to reduce the influence of mechanical contaminations which content, however, in some cases was quite uncontrollable due to holding the whole technological process in normal laboratory conditions. 

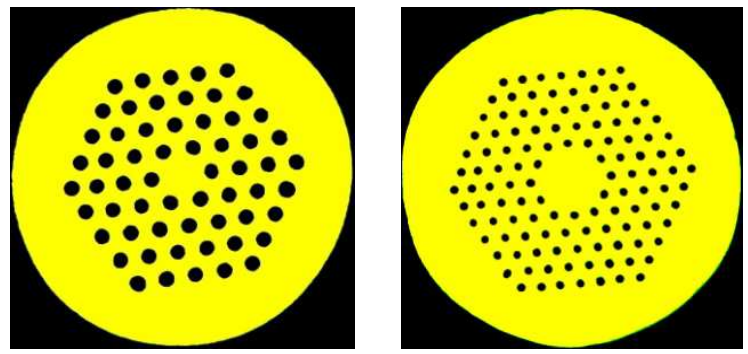

Fig. 2. Microscopic images of 1-element-core (left) and 7-element-core (right) MOF structures.

\subsubsection{Core diameter of $\mathbf{2 0} \boldsymbol{\mu m}$}

We started with the core size that allows a typical MOF to be single-moded over a relatively wide wavelength range in the near infrared part of the spectra. The comparison of attenuation coefficients between the 7-element-core MOF with $\mathrm{k}=0.40$ and the standard 1element-core analog (LMA-20 produced by Crystal Fibre A/S) with $\mathrm{k} \sim 0.49$ is presented in Figure 3. Optical loss measurements were made for a bending diameter of 16 centimeters.

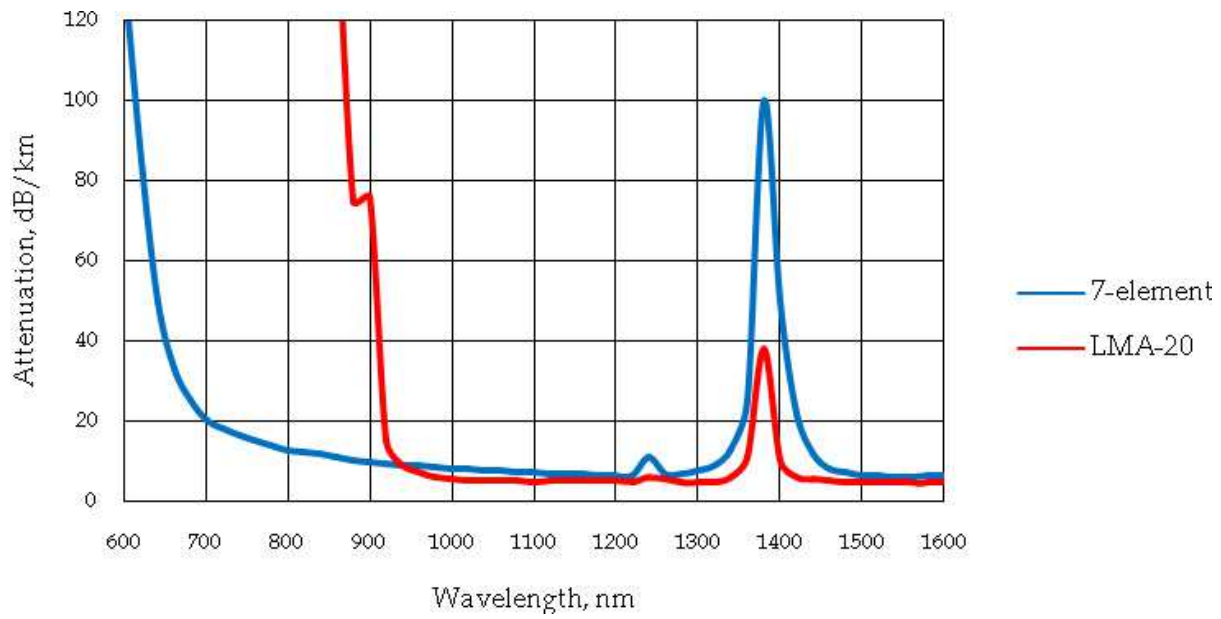

Fig. 3. Spectral attenuation pattern of 7-element-core and 1-element-core (Nielsen et al., 2004b) MOF structures.

Despite the fact that the decrease in the $\Lambda$ value from $13.2 \mu \mathrm{m}$ (Nielsen et al., 2004b) to $6 \mu \mathrm{m}$ in our fiber has a positive effect on the position of bend-loss edge which shifts from 900 to approximately $650 \mathrm{~nm}$, there is no difference in the spectral attenuation behavior between two MOF structures under study. In other words, both curves are smooth and have a low growing tendency while moving to shorter wavelengths. Moreover, they display identically the dramatic increase in attenuation coefficient due to leakage conditioned by the stationary bending radius. Two spectral peaks associated with wavelengths 1250 and $1380 \mathrm{~nm}$ apply naturally to hydroxyl groups absorption. 
We have determined that no higher order mode cut-off can be identified. In the case of conventional single-mode fiber (for instance, SMF-28) higher order mode cut-off appears as an abrupt power decrease in attenuation curve, so far as $\mathrm{LP}_{11}$-mode leaks intensively in the spectral region close to cut-off wavelength. Noted decrease can be described by the exact value of $4.8 \mathrm{~dB}$ (Jeunhomme, 1983) determined by either presence or absence of $\mathrm{LP}_{11}$-mode radiation at the output end of the fiber. The value of $4.8 \mathrm{~dB}$ does not depend on the fiber length and should be observed undoubtedly in the fiber under investigation, since total light attenuation of the measured piece normally does not exceed $10 \mathrm{~dB}$.

A slight decrease in the core size from 20 to $18 \mu \mathrm{m}$ enables an additional absorption peak due to non-bonding oxygen to be clearly revealed at wavelength $\lambda=630 \mathrm{~nm}$ (Figure 4). One may notice further move of the short-wavelength bend-induced leakage boundary to the ultraviolet part of the spectra. The value of k-parameter can also affect the position of macrobending loss edge owing to a greater or lower contrast between the core and the cladding effective refractive indexes. In any case air-filling fraction needs to be controlled accurately because in some cases it may lead to multimode regime of operation.

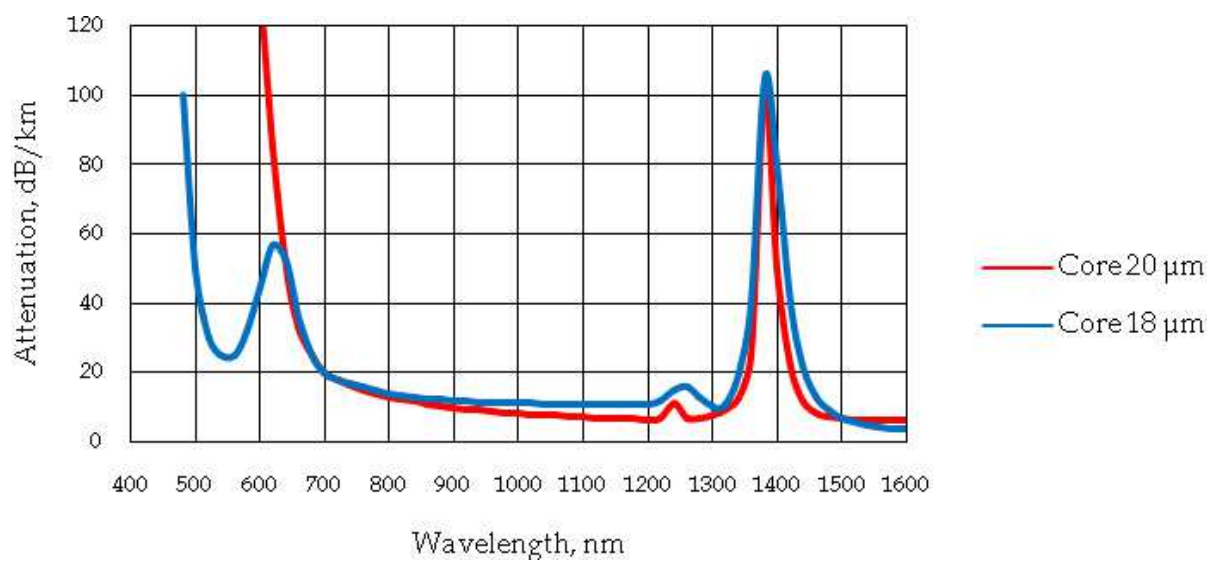

Fig. 4. Dependence of the optical losses on the decrease in the MOF core diameter.

Theoretically, the endlessly single-mode MOF is realized when $\mathrm{k}<0.45$ (Mortensen, 2002) in case the core is represented by one missing air hole. Referred fiber (Nielsen et al., 2004b) performs the lattice structure with $\mathrm{k} \sim 0.49$ that is close enough to the endlessly single-mode regime condition. The fiber is definitely interpreted to be the single-mode one over the entire spectral range studied from $\sim 900$ to $1600 \mathrm{~nm}$ (Figure 3, red curve). So we suppose that the MOF with the 7-element core of $20 \mu \mathrm{m}$ in diameter and k-parameter equal to 0.40 is also single-mode in consequence of the absence of power drops in smooth attenuation spectra from 650 to $1600 \mathrm{~nm}$. Nevertheless, we have investigated roughly modal properties of the fabricated fiber. To this effect, we launched radiation from He-Ne laser $(\lambda=633 \mathrm{~nm})$ into the sample and then observed visually typical Gaussian far-field intensity distribution on the screen placed at the distance of approximately 10 centimeters far from the fiber output. In addition, the rated value of half-divergence angle was equal to several hundredths of a radian that completely corresponded with the fundamental mode operation as well. 
Those first raw results inspired us to carry more profound analysis of the 7-element-core MOF structures.

\subsubsection{Core diameter of $\mathbf{2 5} \boldsymbol{\mu m}$}

To verify the preceding assumption of the single-mode behavior of radiation propagated along the 7-element-core MOF with $\mathrm{k}=0.40$, extra procedures were carried out approaching the opposite to $\lambda=633 \mathrm{~nm}$ part of the spectral range. Those actions were addressed towards a new set of fibers with the core size of $25 \mu \mathrm{m}$ in diameter and k-parameter ranging from 0.19 to 0.50 .

Figure 5 shows the quasi-single-mode character of the spectral attenuation curve regardless of the k-parameter value. All presented spectra do not contain any noticeable peaks of the higher order mode cut-off. Besides that, there exists pronounced short-wavelength leakage boundary specified by the fiber placement on a spool with a diameter of 16 centimeters. One can also see that the position of this boundary depends directly on the k-parameter: the larger the $\mathrm{k}$ value the shorter the wavelength of bend-loss edge. Basic levels of attenuation are defined by various degree of the initial stack purification.
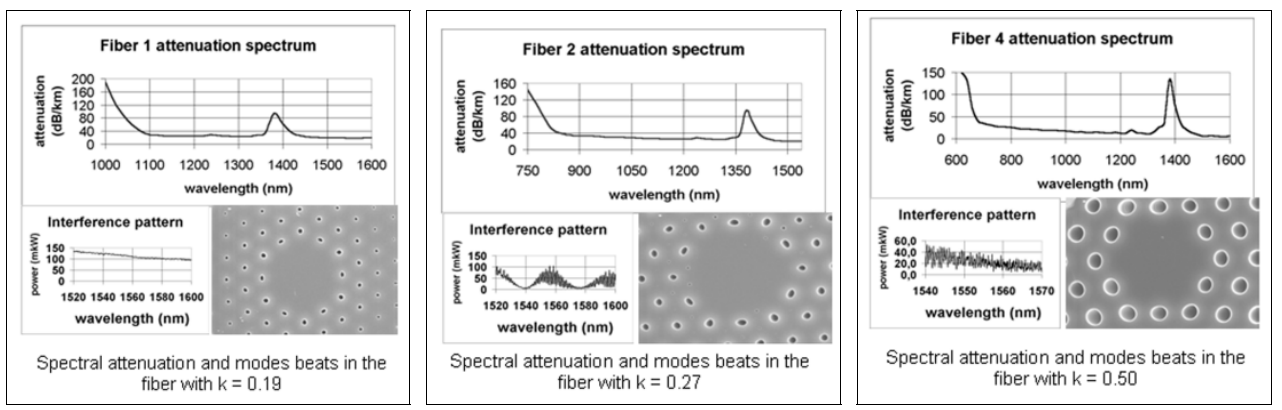

Fig. 5. Dependence of the optical losses on the MOF geometrical parameters.

The fibers, presented in Figure 5, having a length from 20 to 100 meters were investigated by means of the modal beats method. For this purpose, spectrally narrow radiation of tunable semiconductor laser $(\lambda=1520-1580 \mathrm{~nm})$ was launched into a piece of SMF-28 connected with the target MOF sample. The output signal passed through a reciprocal piece of SMF-28 to PT2010 optical power meter, resulting in the modal beats pattern, registered as a change in the power distribution of light. Thus, the output signal could present the beats between two or more guided modes, i.e. intermodal interference (Figure 5, central, right), or the absolute lack of the beats in the single-mode regime (Figure 5, left). We controlled the validity of the modal beats method by comparing two schemes of the experiment in the selected spectral region. The subsidiary technique consisted in visualization of infrared radiation $(\lambda \sim 1550 \mathrm{~nm})$ on a special screen yielding an implicit coincidence with the data given by the main scanning method.

It should be noted that in spite of the smooth character of all spectral attenuation curves, given in Figure 5, and the absence of power drops corresponding to the higher order mode cut-off, the modal beats method has designated clear pattern of modal interaction. Those new results have confirmed the uncertainty of the single-mode behavior of the 7-elementcore structures with the core diameter of $20 \mu \mathrm{m}$, examined in the previous paragraph. 
In the case of excitation of the second order mode the output signal can be described according to the following expression:

$$
I=A^{2}+B^{2}+2 A B \cos \left(\omega \Delta n_{e f f} L / c\right),
$$

where $\mathrm{A}$ and $\mathrm{B}$ are the amplitudes of $\mathrm{LP}_{01}$ and $\mathrm{LP}_{11}$ modes respectively, $\omega$ is the circular frequency, $\Delta \mathrm{n}_{\text {eff }}$ is the difference between effective mode indexes, $\mathrm{L}$ is the sample length and $\mathrm{c}$ is the speed of light in vacuum.

We have applied the discrete Fourier transform and, as a result, have determined the spatial frequencies $\mathrm{v}$ corresponding to the peaks of the interference pattern. Then we have calculated the effective index difference between the fundamental and the excited second order mode by applying formula:

$$
\Delta \mathrm{n}_{\mathrm{eff}}=\mathrm{v} \lambda^{2} / \mathrm{L} .
$$

The experimental data are summarized in Table 1.

\begin{tabular}{|c|c|c|c|c|}
\hline Fiber & k-parameter & Length, $\mathrm{m}$ & $\mathrm{v}, \mathrm{nm}^{-1}$ & $\Delta \mathrm{n}_{\text {eff }}$ \\
\hline 1 & 0.19 & 19 & No beats & - \\
\hline 1a & 0.19 & 0.8 & 0.06 & 0.0002 \\
\hline 2 & 0.27 & 2.0 & 0.8 & 0.00096 \\
\hline 3 & 0.34 & 1.6 & 0.7 & 0.00105 \\
\hline \multirow{2}{*}{$3 \mathrm{a}$} & \multirow{2}{*}{0.34} & 3.2 & 1.5 & 0.0010 \\
& & & 3.2 & 0.0024 \\
\hline & \multirow{3}{*}{4} & \multirow{2}{*}{2.8} & 1.3 & 0.0011 \\
& & & 1.8 & 0.0015 \\
& & & 4.0 & 0.0034 \\
& & & 4.8 & 0.0041 \\
\hline
\end{tabular}

Table 1. Dependence of the effective mode index difference between the fundamental and the second order mode on the MOF structure (Agruzov et al., 2008).

As the experimental data show, vast majority of the MOF samples are characterized by the propagation of at least two guided modes. The value of the effective mode index difference $\Delta \mathrm{n}_{\text {eff }} \sim 0.001$ exists for all presented samples regardless of the k-parameter. Only exception to the general tendency is Fiber 1 with $\mathrm{k} \sim 0.19$, described by the absolute lack of the modal beats even when resolution capacity of registering system is the order of magnitude higher than the common level enough for the clear interference pattern visualization in all other cases. A piece of Fiber 1 having a length of 19 meters is single-moded, while a shorter piece of the same fiber (Fiber 1a) demonstrates the presence of the second order mode at the output end. So the higher order mode can be characterized by the essentially greater attenuation coefficient than the fundamental one.

Further investigations aimed at the determination of the spectral operation width of Fiber 1. But the attempt to obtain the pattern of far-field intensity distribution utilizing the available light source (He-Ne laser) failed: laser radiation intensively leaked away from the core area and filled the entire cladding even in the piece of about 1 meter in length. All other fibers from the list of Table 1 have demonstrated the modal interference pattern at $\lambda=633 \mathrm{~nm}$ : power distribution of light depended on the input fiber geometry and on the conditions of 
light propagation along the MOF. A bend or a mechanical stress influenced definitely the far-field pattern so the intensity peak moved from one part of the spot to another.

Reverting to the subject of the absence of $\mathrm{LP}_{11}$-mode cut-off in the spectral attenuation patterns (Figures 3,5), we should state that the higher order mode (or modes) exists simultaneously with the fundamental one in the spectral interval $\lambda=600-1600 \mathrm{~nm}$. Normalized frequency $\mathrm{V}$ is weakly dependent on wave number. That fact differs MOFs from other types of lightguides. So for the determined wavelength range V-parameter varies negligibly (Mortensen et al., 2003), preserving its magnitude almost invariable (Figure 6). Since V-parameter directly defines the amount of guided modes, a number of them can coexist persistently within a spectral range, specific for each fiber, and at the same time undergo the infinite attenuation by reason of the huge power leakage at the identical wavelength in the blue part of the spectra.

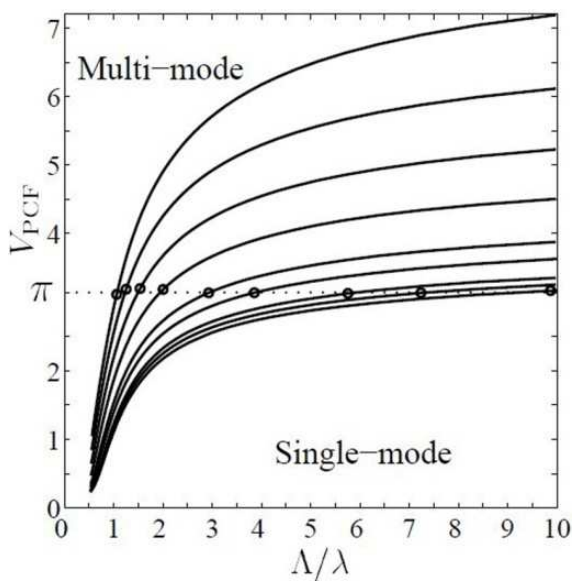

Fig. 6. Dependence of the normalized frequency V on the MOF structure (Mortensen et al., 2003). $V_{P C F}=\Pi$ is the cut-off condition for the second order mode.

Finally, we have accurately determined that the single-mode operation can be achieved by the suitable selection of the k-parameter (Figure 5). The reduction of k-parameter causes the move of bend-induced leakage boundary from $\lambda \sim 650$ to $\lambda \sim 1000 \mathrm{~nm}$. Unfortunately, there is no preference of the 7-element-core MOF design over the standard 1-element-core analog (LMA-25 produced by Crystal Fibre A/S): both fibers have a bend-loss edge located in the wavelength region of $1 \mu \mathrm{m}$ while being bent on a spool of $16 \mathrm{~cm}$ in diameter.

\subsubsection{Core diameter of $\mathbf{3 5} \boldsymbol{\mu m}$}

The improved situation takes place in case of the further core enlargement approaching 35 $\mu \mathrm{m}$. It is a well-known fact that such great leap in the core diameter strongly affects the width of the spectral operation range (Nielsen et al., 2004b) and actually transforms the fiber into a 'single-frequency' optical element which is operable exclusively at $\lambda=1550 \mathrm{~nm}$. We have yielded some positive results in development of the bend-resistant MOF design having a core of $35 \mu \mathrm{m}$ in diameter.

There are three fiber samples, presented in Table 2, made of the same initial capillary stack with the air-filling fraction variable within the range $\mathrm{k}=0.2-0.4$. The experimental data 
displays the way how the reduction of k-parameter influenced the modal properties of light propagated. When $\mathrm{k}$ was about 0.4 the fiber was the multimode one both in red and infrared parts of the spectra. As the value of k-parameter decreased approximately twice, the fiber turned into the single-mode one, at first, in the visible part of the spectra and then in the infrared part.

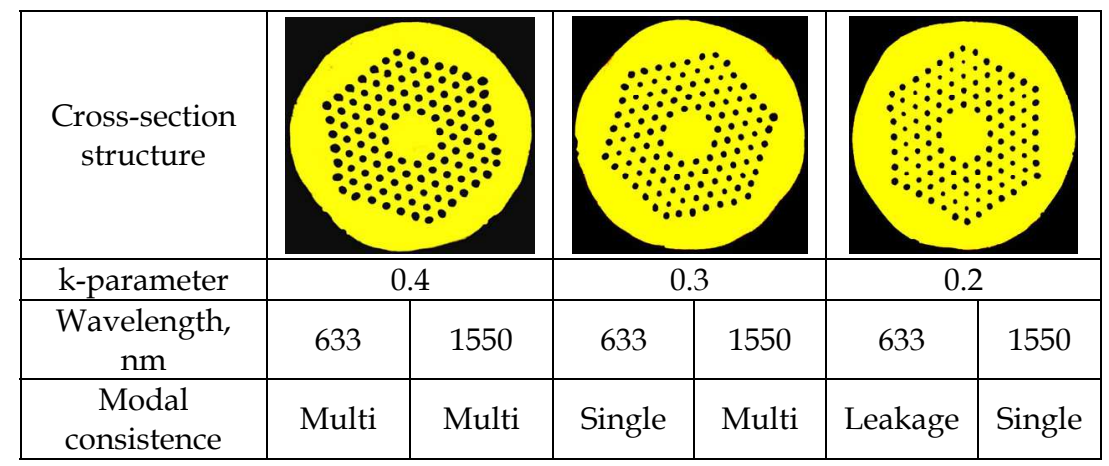

Table 2. Dependence of the modal properties on the k-parameter reduction.

Additional investigations of the fundamental mode spot size were carried out. For this purpose, conventional single-mode fiber with the core diameter of $8 \mu \mathrm{m}$ was attached to the MOF. Under mutual end-facet scanning the Gaussian-like power distribution of light was obtained. The MOF was excited by laser radiation at $\lambda=1550 \mathrm{~nm}$. The results have shown that the mode spot size in the 7-element core structure is about $26 \mu \mathrm{m}$ that corresponds equally to the 1-element core analog (Nielsen et al., 2003) despite the difference both in the amount of air holes, surrounding the core area (12 or 6), and in the k-parameter values (0.19 or $\sim 0.49$ ).

It is necessary to note that all presented MOF structures are not strongly single-mode ones if you keep in mind the existence of inherent to MOFs the endlessly single-mode regime of light propagation. For the 7-element-core microstructure this regime is provided at $\mathrm{k}<0.046$ (Saitoh et al., 2005). It is clear that the structure with such low value of k-parameter cannot be correctly realized in practice. Nevertheless, the single-mode operation can be carried out due to the significant difference in attenuation coefficients of the fundamental and the higher order modes.

For the benefit of such point of view, the behavior of the modal properties dependent on the exact k-parameter value testified (Table 2). As it may be seen, for the $k=0.3$ in the red part of the spectra there existed only the fundamental mode, though in the infrared part we have observed several modes. This situation cannot be explained otherwise than by the strong attenuation coefficient of the higher order modes. In the case of conventional fiber made of the materials with different refractive indexes we observe the opposite tendency according to the expression (Snyder \& Love, 1983):

$$
\mathrm{V}=2 \pi \mathrm{a} \sqrt{\mathrm{n}_{1}^{2}-\mathrm{n}_{2}^{2}} / \lambda,
$$

where $\mathrm{a}$ is the core radius, $\mathrm{n}_{1}$ and $\mathrm{n}_{2}$ are the core and the cladding refractive indexes respectively. The decrease of normalized frequency $\mathrm{V}$ causes the reduction of the amount of 
excited modes, so in the red part there would be several modes and in the infrared part only the fundamental one. This dependence can also be retained in the case of MOFs with correction for the effective values of the refractive index (Russell, 2006).

Finally, we declare that the higher order mode undergoes considerably strong attenuation in the visible part of the spectra than the fundamental one. This assertion has the corresponding interpretation: the shorter the wavelength the larger divergence due to diffraction and/or leakage of the higher order mode into the gaps between the air holes (Russell, 2006). By means of varying $\mathrm{k}$ and $\Lambda$ parameters it is possible to fit the proper conditions in which the fundamental mode attenuates slightly in comparison with the higher order mode. Here we must say that the latest statement is correct only for the determined wavelength range.

Spectral attenuation pattern (Figure 7) shows the obvious preference of the 7-element-core MOF design over the standard 1-element-core analog (LMA-35 produced by Crystal Fibre A/S): the position of bend-induced leakage boundary moves to the blue part of the spectra for about $100 \mathrm{~nm}$. Comparison is considered for a bending diameter of 32 centimeters.

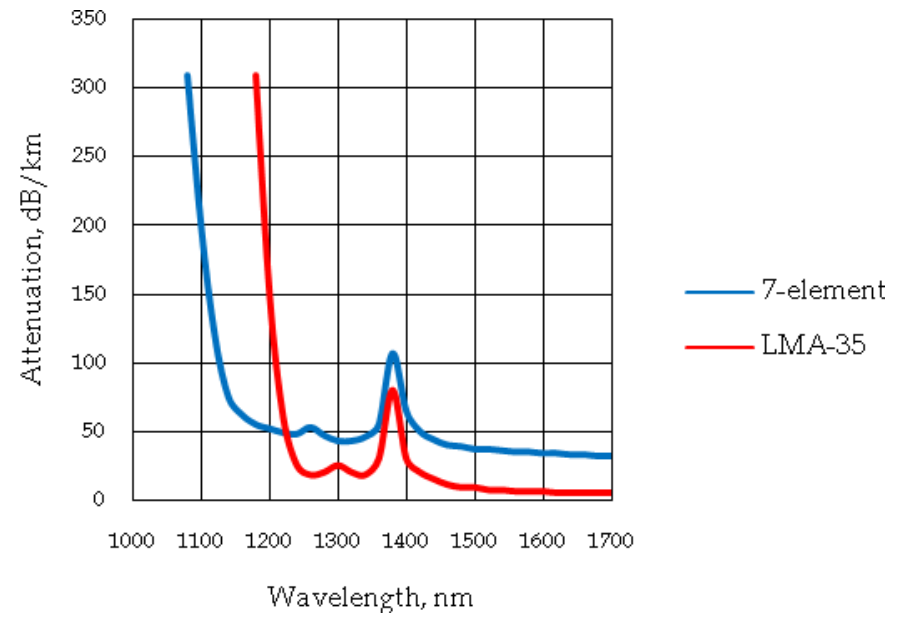

Fig. 7. Spectral attenuation pattern of 7-element-core and 1-element-core (Nielsen et al., 2004b) MOF structures.

\subsection{MOFs with the core comprised of 19 missing holes}

Even more impressive results have been obtained in the development of the MOFs with the core area formed by the initial substitution of 19 central capillaries in the original stack for one solid rod (Figure 8).

We have observed the similar situation for the 19-element-core MOFs with $\mathrm{k}=0.3$ as for the MOFs discussed in previous paragraph. If look carefully at the family of the spectral attenuation curves, presented in Figure 9, one can note that the increase of the core diameter leads directly to the shift of bend-induced leakage boundary of the fundamental mode to longer wavelengths within the spectral range explored. Also there is no evidence of the higher order mode cut-off in attenuation curves. All the fibers turned out to be the singlemode ones with the appreciable preference in the spectral operation range widening over the 7-element-core MOF structures and, what is more, over the 1-element-core analogs. 


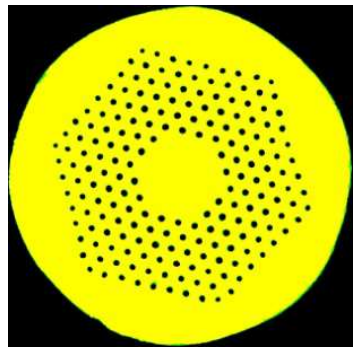

Fig. 8. Microscopic image of the 19-element-core MOF structure.

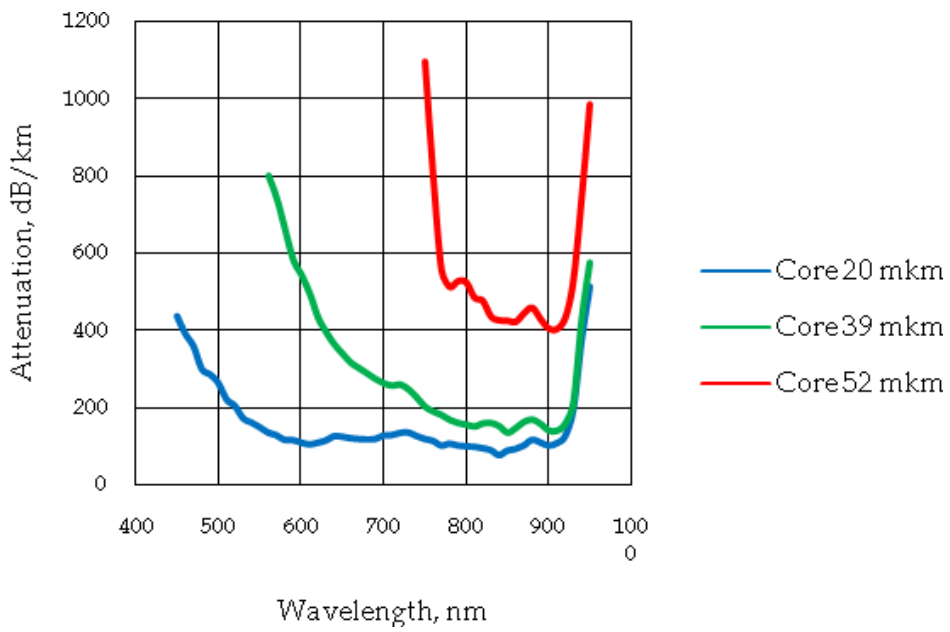

Fig. 9. Spectral attenuation pattern of the 19-element-core MOFs.

At the same time, solid analysis of the modal consistence of the 19-element-core MOFs have shown the complicated behavior of the higher order mode (Table 3).

\begin{tabular}{|c|c|c|c|c|c|c|c|c|}
\hline \multirow{2}{*}{ Fiber } & \multirow{2}{*}{$\begin{array}{c}\text { Core } \\
\text { diameter, } \\
\mu \mathrm{m}\end{array}$} & \multirow{2}{*}{$\begin{array}{c}\text { Short- } \\
\text { wavelength } \\
\text { leakage } \\
\text { boundary, nm } \\
\text { (Spool diameter } \\
16 \mathrm{~cm})\end{array}$} & \multirow{2}{*}{ Length, $\mathrm{m}$} & \multirow{2}{*}{$\begin{array}{c}\mathrm{k}- \\
\text { parameter }\end{array}$} & \multicolumn{2}{|c|}{$\begin{array}{l}\text { Spool diameter } \\
16 \mathrm{~cm}\end{array}$} & \multicolumn{2}{|c|}{$\begin{array}{c}\text { Spool diameter } \\
32 \mathrm{~cm}\end{array}$} \\
\hline & & & & & $633 \mathrm{~nm}$ & $\begin{array}{c}1550 \\
\mathrm{~nm}\end{array}$ & $633 \mathrm{~nm}$ & $\begin{array}{l}1550 \\
\mathrm{~nm}\end{array}$ \\
\hline 1 & 45 & 1200 & 6 & $\sim 0.2$ & Leak. & $\begin{array}{c}2 \\
\text { modes }\end{array}$ & \multirow{4}{*}{\multicolumn{2}{|c|}{2 modes }} \\
\hline 2.1 & 27 & 600 & 2.5 & $<0.2$ & $\begin{array}{c}2 \\
\text { modes }\end{array}$ & $\begin{array}{c}2 \\
\text { modes }\end{array}$ & & \\
\hline 2.2 & 27 & 600 & 10 & $<0.2$ & 1 mode & 1 mode & & \\
\hline 2.3 & 27 & - & 25 & $<0.2$ & 1 mode & 1 mode & & \\
\hline
\end{tabular}

Table 3. Dependence of the modal properties of the 19-element-core structure on the MOF geometrical parameters (Agruzov et al., 2010). 
In fact, all presented samples are the multimode ones in case of straight fiber segments so far as the 19-element core guides a few higher-order transverse modes under $k>0.2$. However, both the controllable reduction of k-parameter and the fiber placement on a spool decrease the amount of the excited modes. The single-mode behavior of the MOF bent on a spool of 16 centimeters in diameter states stationary at the fiber lengths of more than 10 meters. Then the fiber demonstrates the improved bending resistance properties and hence excellent leakage characteristic. At shorter lengths the presence of the higher order mode radiation at the output end of the fiber is unavoidable.

Particularly, Figure 10 illustrates how successfully the $\Lambda$-parameter reduction can be carried out in the 19-element-core MOF (Fiber 2.2).

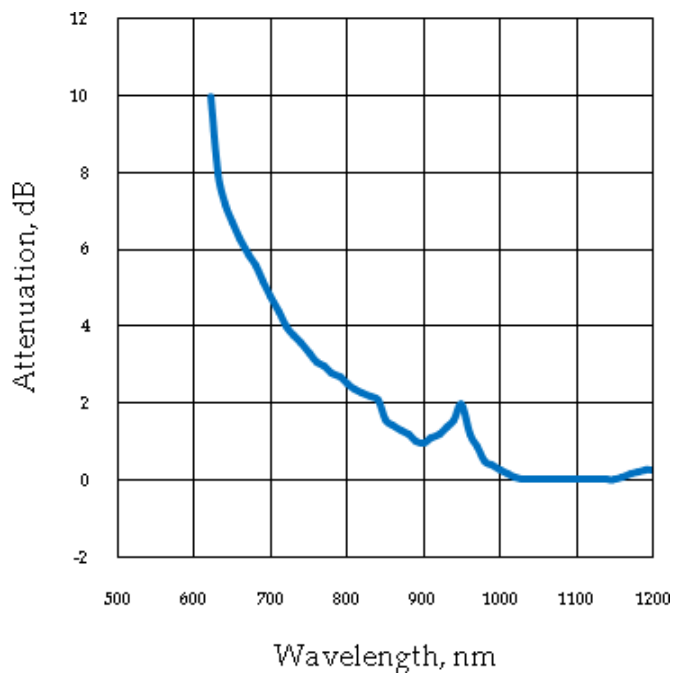

Fig. 10. Spectral attenuation pattern of Fiber $2.2(\mathrm{k}<0.2)$.

The position of bend-loss edge is located in the red part of the spectra even when the fiber is placed on a spool of 16 centimeters in diameter. It is rather difficult to compare accurately how far the features of the 19-element-core MOF vary from the standard 1-element-core analog because of the lack of literary data. In any case the leakage characteristic is better than that of the 1-element core of $20 \mu \mathrm{m}$ in diameter (Nielsen et al., 2004b), where optical losses increase dramatically at the wavelength $\lambda \sim 900 \mathrm{~nm}$. As in the case of the 7-elementcore MOF, the single-mode propagation takes place when the k-parameter is equal or less than 0.2 . For the larger $\mathrm{k}$ values the fiber definitely becomes the multimode one.

Our investigations (not included in Table 3) also have shown that straight pieces of Fiber 2.2 and Fiber 2.3 support several transverse modes at the lengths available in normal laboratory areas (up to 10 meters). This circumstance restricts the application potential of the 19element-core MOFs, especially if it is necessary to obtain guaranteed single-mode regime. For example, this may take place in high power beam delivery systems, where radiation is passed from the stationary placed laser to the variable operation area. In such situation the modal consistence strongly depends on the fiber configuration. On the other hand, if one needs to transport the energy through the multibend sleeve of the fiber placed in production areas of complicated architecture, the priority of the 19-element-core MOF is evident. 


\subsection{Special attenuation mechanism}

While investigating the modal properties of the aforementioned MOF structures with the large 7- or 19-element core, we have defined a specific mechanism when the attenuation coefficient of the higher order mode substantially exceeds the same parameter of the fundamental one. This phenomenon had the most decisive effect on the modal consistence of the MOFs with the core of $35 \mu \mathrm{m}$ in diameter, allowing the fibers with $\mathrm{k}<0.2$, being bent on a standard spool with a bending diameter of 16 centimeters, to propagate only the fundamental mode within a broad spectral range $\lambda=600-1550 \mathrm{~nm}$. Draw attention that, theoretically, the higher order mode cut-off condition for this fiber is expected to be technologically unfeasible (since for the 7-element-core fiber k-parameter, in theory, has to be as low as 0.046 to ensure the single-mode operation). So the great difference in attenuation coefficients of $\mathrm{LP}_{01}$ and $\mathrm{LP}_{11}$ modes enables the implementation of the 19element-core MOF with the absolutely workable $\mathrm{k} \sim 0.2$, operating in the single-mode regime (Fiber 2.2 and Fiber 2.3).

To determine the proportion of the attenuation coefficients, sufficient for the single-mode operation via the differential modal attenuation, we have estimated the optical losses of the higher order mode. For this purpose, we have measured the depth of modulation while registering several patterns of the modal beats. The length of the investigated Fiber 2.2 sample was varied deliberately to achieve distinct patterns ( 1 meter, 1.5 meters and 2 meters). This procedure has shown the inverse influence of the fiber length on the depth of modulation: less pronounced pattern corresponded to larger sample lengths. Thus, the attenuation coefficient of the higher order mode was evaluated to be $\sim 5 \mathrm{~dB} / \mathrm{m}$. At the same time, the fundamental mode attenuation coefficient, measured by a cut-back technique, have been rated at a level of tens of $\mathrm{dB} / \mathrm{km}$. So now we can state that the fundamental mode propagation via the differential modal can be effectively realized in MOF structures when the higher order mode attenuation coefficient is of at least two orders of magnitude larger than the analogous parameter of the fundamental mode.

The next goal consisted in detailed analysis of the conditions opportune enough for the establishment of the differential modal attenuation. The implementation of microstructures with large cores, irrelevant to 7 or 19 elements, and great $\Lambda$-parameter values seemed to be the most applicable means to collect the data.

\section{MOFs based on the differential modal attenuation mechanism}

In the previous part of the work the special attenuation mechanism has been shown. It described the situation when the attenuation coefficient of the fundamental mode may be essentially lower than the same optical parameter of the higher order mode. Starting from that point, we have concentrated our efforts on the extensive research of a few novel MOF designs that could successfully correspond with a set of special requirements: appreciable difference in optical losses of the first two modes $\left(\mathrm{LP}_{01}\right.$ and $\left.\mathrm{LP}_{11}\right)$, single-mode operation, high bending resistance and fiber placement on a standard transport spool of 16 centimeters in diameter. Among the others we have tested structures with the circular cladding distribution, with the special $C_{3 V}$ cladding symmetry and with the 1-element shifted core. All of these MOF structures seemed to fit with the requirements, especially with the enforcement of the higher order mode to undergo the enhanced attenuation. We can say in advance that the differential modal attenuation mechanism better displays in case of the core diameters of more than $30 \mu \mathrm{m}$ and the k-parameters as large as 0.60 . 


\subsection{Investigation procedures}

In order to investigate carefully the modal properties of the MOFs as a function of the transverse fiber dimensions, we used the layout: a set of semiconductor lasers $(\lambda=658,808$, 980 and $1550 \mathrm{~nm}$ ), objective lenses, micrometer screws, digital CCD-camera (640 x 480 with the pixel size of $7 \mu \mathrm{m}$ ), fiber cutter and personal computer for data handling. We have been registering near-field or far-field patterns of the radiation propagated along the fibers while varying the input coupling conditions. For this purpose, laser radiation was launched into the test fiber under diverse apertures to achieve the most powerful signal on a CCD-camera screen. The fundamental mode specified by the Gaussian power distribution of light was the easiest to excite. If under varying the input coupling geometry we observed only the fundamental mode alteration (the modal spot became larger or smaller uniformly) and the higher order mode did not appear at the output end of the fiber, we explicitly considered the situation to be the single-mode one (Figure 11, left). The amount of modes in tables below was noted as 1 . In the other case, when we clearly ascertained the distortion of the Gaussian power distribution or mode superposition with nearly equivalent peak power levels (Figure 11, right), we denoted the amount of the excited modes as 2.

In addition, we have made the evaluation of the mode spot size of the fiber samples with the definite single-mode regime of operation. We have modified the well-known expression for the half-divergence angle (Mortensen et al., 2002) to the form:

$$
\omega=\lambda \mathrm{L} / \Pi \mathrm{W},
$$

where $\mathrm{W}$ is the mode spot size measured at $1 / \mathrm{e}^{2}$ level of peak intensity on the CCD-camera screen and $\mathrm{L}$ is the distance between the screen and the fiber end-facet.
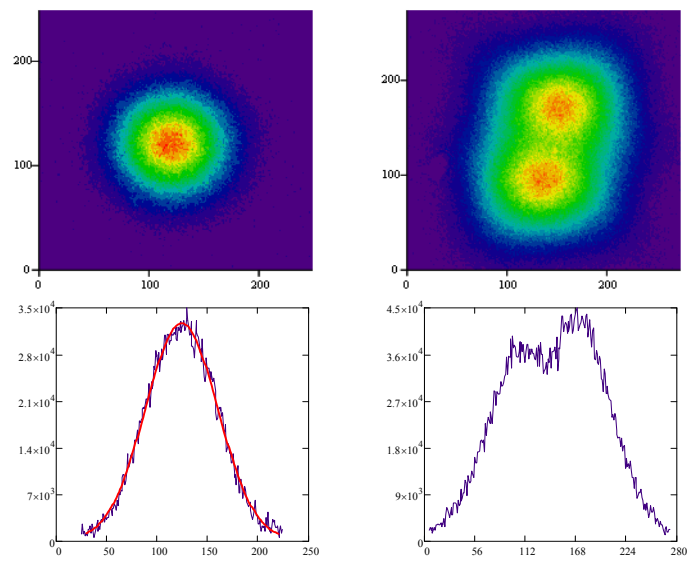

Fig. 11. Far-field patterns of the MOFs: fundamental mode propagation with the Gaussian approximation (left) and mode superposition (right).

\subsection{MOFs with the circular cladding distribution}

We have designed and manufactured a novel type of silica-based MOF containing a solid glass core of $30 \mu \mathrm{m}$ in diameter and three air-hole rings organizing light-reflecting cladding, as it is shown in Figure 12. The similar structure containing 8 circles was mentioned in (Martelli et al., 2007) 


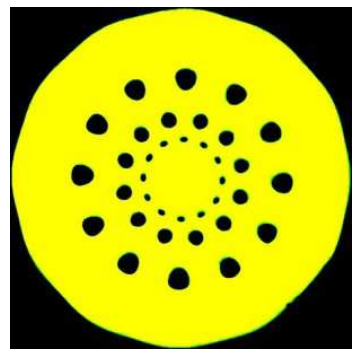

Fig. 12. The MOF structure with the circular cladding distribution.

The principal difference between the standard triangular MOF structure and the circular one is that in the latter case the amount of air-filled channels does not increase within next ring but remains invariable while going from the core area to the outer fiber boundary. In whole, the number of air holes in each ring has to be relatively large to provide a great contrast between the core refractive index and the effective cladding refractive index. In our case the number of air holes in each of the successive rings surrounding the core area was 12, which, in our opinion, is large enough to guarantee satisfactory bending characteristics. On the other hand, a quite large number of air holes will ensure good light confinement in the core area. Another important feature, worthy of attention, is the shape of the fundamental mode spot. So far as the air holes distribution in the first ring replicates the form of nearly a circle, the MOF structure performs the circular-like shape of the modal spot $\left(\mathrm{C}_{12 \mathrm{~V}}\right.$ symmetry), that in some cases may be more preferable as compared to a typical six-fold rotational one ( $\mathrm{C}_{6 \mathrm{~V}}$ symmetry). As it is illustrated in Figure 12, at first, we have tended to equalize the k-parameter value in the air-hole rings, because in the triangular lattice that parameter, actually, is approximately constant over the MOF cross-section. In order to fabricate this structure we used three sets of the initial capillaries made of quartz glass. They were characterized by the outer diameters of $1.25,2.10$ and 3.30 millimeters. The ratio of the inner to the outer diameters in all three sets of capillaries was equal to $\sim 0.50$.

We have managed to achieve the single-mode operation over the entire spectral range studied $(\lambda=658-1550 \mathrm{~nm})$ in the derived structure, though the attenuation coefficient was too large in consequence of the tremendous leakage of the fundamental mode power into the fiber curve (standard spool of 16 centimeters in diameter). The weak dependence of the mode spot diameter on the wavelength confirms that process (Figure 13).
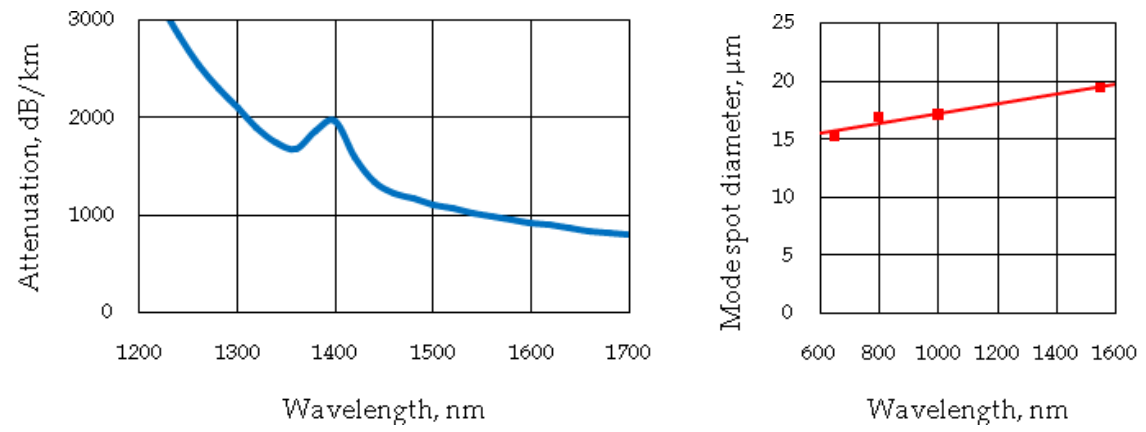

Fig. 13. Spectral attenuation pattern (left) and mode spot diameter (right) of the first series of the MOFs with the circular cladding distribution. 
The sensitivity of the propagated radiation to bend may be explained largely by its great leakage through the silica gaps between the air holes in the external ring.

In the second and third series of MOFs with the circular cladding distribution we deliberately used the initial capillaries with the increased ratio of the inner to the outer diameters (Table 4).

\begin{tabular}{|c|c|c|}
\hline Air-hole ring & Ratio in the second series & Ratio in the third series \\
\hline 1 (nearest to the core area) & 0.8 & 0.8 \\
\hline 2 & 0.75 & 0.8 \\
\hline 3 & 0.75 & 0.8 \\
\hline
\end{tabular}

Table 4. Inner/outer diameters ratio for the circular-cladding structures.

Using the capillaries with the geometrical parameters presented in Table 4 we have obtained tangible reduction in the optical losses of the fundamental mode. Moreover, there was no evidence of the second order mode radiation both in 3-meter-length and 30-meter-length samples. The leakage losses of the $\mathrm{LP}_{11}$ mode have been estimated to be about $10 \mathrm{~dB} / \mathrm{m}$. The experimental results are accumulated in Table 5.

\begin{tabular}{|c|c|c|c|c|}
\hline \multirow{2}{*}{$\begin{array}{c}\text { Core } \\
\text { diameter } \\
\mu \mathrm{m}\end{array}$} & \multicolumn{4}{|c|}{ Modal consistence } \\
\end{tabular}

Table 5. Modal consistence of the second $(30 \mu \mathrm{m})$ and the third $(40 \mu \mathrm{m})$ series of the MOFs with the circular cladding distribution.
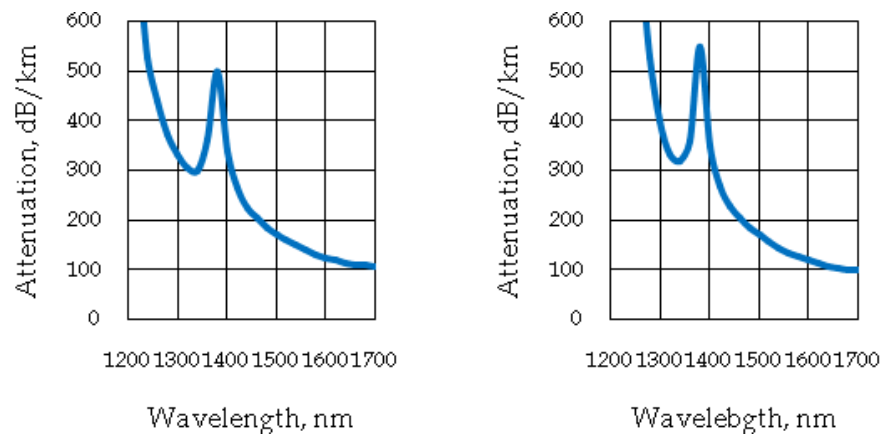

Fig. 14. Spectral attenuation pattern of the MOFs with the circular cladding distribution: the core diameters are $30 \mu \mathrm{m}$ (left) and $40 \mu \mathrm{m}$ (right). 
As Figure 14 illustrates, the MOF with the core diameter of $30 \mu \mathrm{m}$ has excessively large optical losses. However, a certain increase in the air-filling fraction in each of three air-hole rings and simultaneous enlargement of the core size (up to $40 \mu \mathrm{m}$ ) give the structure optimal correlation between high attenuation coefficient of the upper modes and reasonable bend performance of the fundamental mode. Table 5 shows that the MOF with the core diameter of $40 \mu \mathrm{m}$ is characterized by the relatively large mode spot size, ranging from 25.7 to $31.5 \mu \mathrm{m}$ in the inspected spectral range. Despite the significant attenuation coefficient, about 0.15 $\mathrm{dB} / \mathrm{m}$ at $\lambda=1550 \mathrm{~nm}$, the MOF is operable for a standard bending diameter of 16 centimeters. In identical conditions, a well-known triangular structure with the 1-element core undergoes the infinite attenuation (Nielsen et al., 2004b).

We assert that the considerable reduction in the attenuation coefficient of the MOFs with the circular cladding distribution can be achieved by both proper variation of the air-hole sizes and appropriate adjustment of the silica gaps between them. Moreover, the presence or the absence of the higher order mode at the output end of the fiber is determined mainly by the air content in the first air-hole ring. At the same time, the air-filling fractions of the second and the third rings, especially the external, define the leakage loss level of the fundamental mode. As additional experiment has shown, the minimal attenuation in the multimode regime (Figure 15) was measured to be $\sim 8 \mathrm{~dB} / \mathrm{km}$ caused mainly by impurities. This MOF has a potential to be used in high-NA applications.

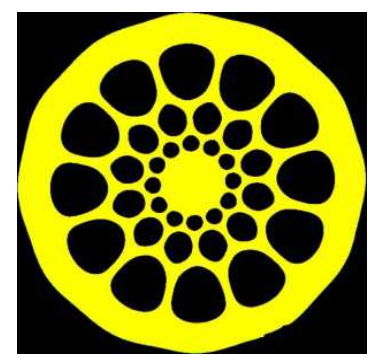

Fig. 15. Microscopic image of the multimode MOF structure with the circular cladding distribution.

\subsection{MOFs with the $\mathrm{C}_{3 \mathrm{~V}}$ cladding symmetry}

We have also fabricated a series of the MOF structures that can be treated as an adaptation of standard 1-element-core design with a triangular arrangement of air holes (Russell, 2006). A key variation consisted in the intentional introduction of an alternation of large and small air holes to the MOF design attaining so called $C_{3 V}$ transverse symmetry (Figure 16), as opposed to the standard $\mathrm{C}_{6 \mathrm{~V}}$ symmetry.
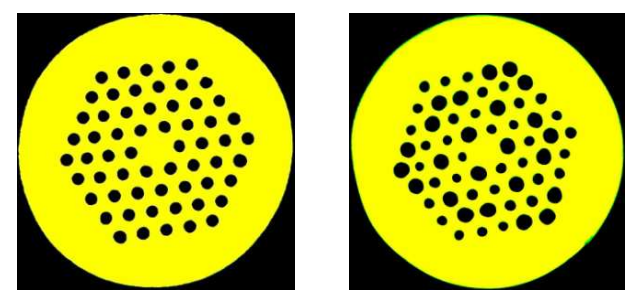

Fig. 16. Microscopic images of the MOFs: typical $\mathrm{C}_{6 \mathrm{~V}}$ symmetry (left) and non-standard $\mathrm{C}_{3 \mathrm{~V}}$ symmetry. 
The alternation of large and small air holes, especially in the air-hole ring closest to the core area, may confidently lead to the enhancement of the higher order mode attenuation. The main mechanism remained the same we had applied earlier for the structure with the circular cladding distribution. We talk about the controllable leakage of the second order mode power through the silica gaps between each pair of neighboring holes.

To achieve the single-mode operation in presented MOF structures we used quartz capillaries of the same outer diameter $(1.75 \mu \mathrm{m})$, but of the diverse inner diameter. The exact ratios of the inner/outer diameters were 0.6 and 0.8. Additionally, we controlled the airfilling fraction during the fiber drawing process by varying two essential technological parameters we could easily change. We mean the capillary pressure and the drawing temperature. The experimental results are given in Table 6. Optical measurements were made for a bending diameter of 16 centimeters.

\begin{tabular}{|c|c|c|c|c|c|}
\hline \multirow{2}{*}{$\begin{array}{c}\text { Core } \\
\text { diameter, } \\
\mu \mathrm{m}\end{array}$} & \multicolumn{4}{|c|}{$\begin{array}{c}\text { Modal consistence } \\
\text { (Fundamental mode spot diameter, } \mu \mathrm{m})\end{array}$} & \multirow[t]{2}{*}{ Cross-section } \\
\hline & $\lambda=658 \mathrm{~nm}$ & $\lambda=808 \mathrm{~nm}$ & $\lambda=980 \mathrm{~nm}$ & $\lambda=1550 \mathrm{~nm}$ & \\
\hline 25 & Leakage & Leakage & $\begin{array}{c}1 \\
(16.8)\end{array}$ & $\begin{array}{c}1 \\
(19.6)\end{array}$ & \\
\hline 35 & Leakage & Leakage & Leakage & $\begin{array}{c}1 \\
(24.4)\end{array}$ & \\
\hline
\end{tabular}

Table 6. Modal consistence of the MOFs with the $\mathrm{C}_{3 \mathrm{~V}}$ cladding symmetry.
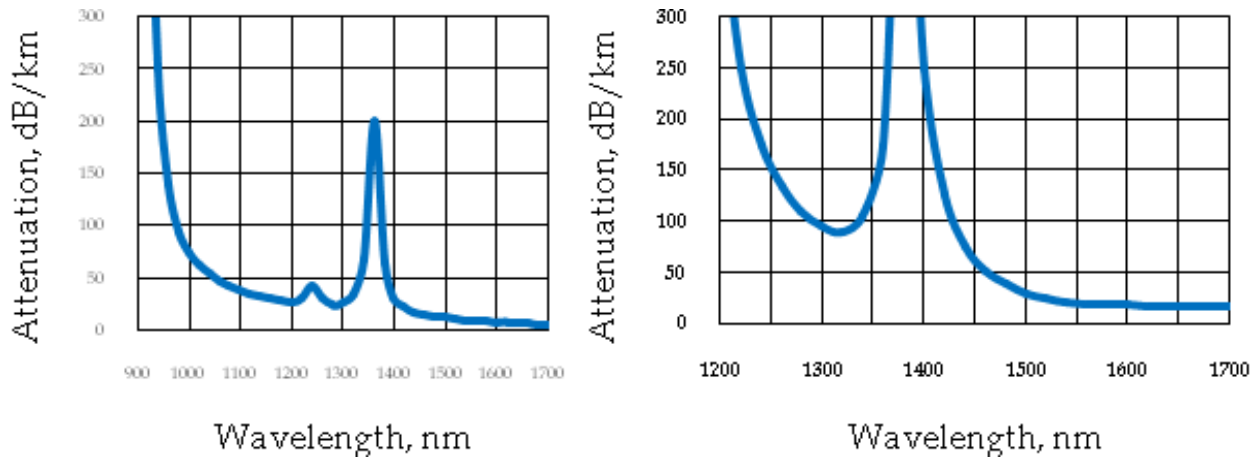

Fig. 17. Spectral attenuation pattern of the MOFs with the $\mathrm{C}_{3 \mathrm{~V}}$ cladding symmetry: the core diameters are $25 \mu \mathrm{m}$ (left) and $35 \mu \mathrm{m}$ (right).

We have detected some positive features of the MOFs with the $C_{3 v}$ cladding symmetry including single-mode regime and high bending resistance properties. The most impressive situation takes place in the fiber with the core diameter of $35 \mu \mathrm{m}$, as the spectral operation 
range can be expanded for about $300 \mathrm{~nm}$ to the blue part of the spectra in comparison with the MOF of a typical triangular configuration with the air holes of invariable dimensions. Classical MOFs are still required to be placed on non-standard spools having a diameter of 32 centimeters (Nielsen et al., 2004b), while our fibers are operable for a bending diameter of 16 centimeters. In addition, as opposed to the MOFs with the circular cladding distribution (discussed in previous paragraph), the fibers with the $\mathrm{C}_{3 \mathrm{~V}}$ cladding symmetry can guarantee optical losses of less than $20 \mathrm{~dB} / \mathrm{km}$ at $\lambda=1550 \mathrm{~nm}$ (Figure 17).

\subsection{MOFs with the shifted core}

This paragraph will cover in detail main optical properties as well as bend characteristics of another MOF structure based on the declared concept of the differential modal attenuation.

Theoretically, the higher order mode of a typical MOF with triangular arrangement of air holes, as opposed to the fundamental mode, may be characterized by a significantly larger degree of the power penetration into the fiber cladding (Tsuchida et al., 2005; Russell, 2006). Applying this specific mode feature, we made an assumption that the MOF structure with the core shifted for the pitch value from its usual location in the center of the lattice structure will exhibit enhanced leakage losses of the higher order mode. At the same time, the leakage losses of the fundamental mode will maintain their value as if the fiber structure is completely retained. So we can implement the special MOF design that enables a relatively simple control of the higher order mode attenuation. A conditioning factor, as in the previous cases we have already studied (MOF structures with the circular cladding distribution and the $\mathrm{C}_{3 \mathrm{~V}}$ cladding symmetry), is the reasonable balance towards the dimensions of the air holes, especially in the ring closest to the core area, and the spaces between them filled with a glass material. We have successfully produced by classical stackand-draw technique the following MOF structure (Figure 18).
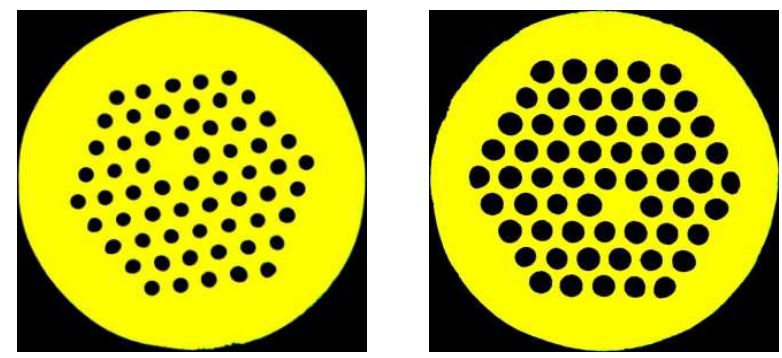

Fig. 18. Microscopic images of the fabricated MOF structures with the shifted 1-element core: $\mathrm{k}=0.45$ (left), $\mathrm{k}=0.75$ (right).

When the core is displaced from the center of the lattice, the peripheral part of the effective $\mathrm{LP}_{11}$-mode area may intensively leak away into the outer fiber cladding which is then situated nearer than in the MOF of a standard central configuration. Thus, the fundamental mode operation is not strongly defined by the phase cut-off condition, known as $\mathrm{k}<0.45$ for the second order mode (Mortensen, 2002). As we will show further, the endlessly singlemode regime may be carried out at certain lengths of the fiber even when the k-parameter value is for more than $40 \%$ larger relative to its theoretical value. In this case the $\Lambda$ parameter and the curve diameter resulting in the bend performance are crucial for the establishment of the differential mode attenuation. 
The experimental results are collected in Table 7. It should be noted that we intentionally fabricated several samples with the standard central-core design to compare two different transverse structures. All optical measurements were made for a bending diameter of 16 centimeters.

One can see from Table 7 that the core diameter enlargement from 12.5 to $20 \mu \mathrm{m}$ under the $\mathrm{k}$-parameter being nearly constant $(\mathrm{k} \sim 0.50)$ caused the additive attenuation of the higher order mode (samples $2_{\text {SHIFT }}$ and $3_{\text {SHIFT) }}$ ).

\begin{tabular}{|c|c|c|c|c|c|c|c|}
\hline \multirow{2}{*}{ Fiber } & \multirow{2}{*}{$\begin{array}{c}\text { Core } \\
\text { diameter, } \\
\end{array}$} & \multirow{2}{*}{$\mathrm{k}$} & \multirow{2}{*}{$\begin{array}{c}\text { Length, } \\
\mathrm{m}\end{array}$} & & \multicolumn{4}{|c|}{$\begin{array}{c}\text { Modal consistence at } \lambda \\
\text { (Fundamental mode spot size, } \mu \mathrm{m})\end{array}$} \\
\cline { 5 - 8 } & & & $658 \mathrm{~nm}$ & $808 \mathrm{~nm}$ & $980 \mathrm{~nm}$ & $1550 \mathrm{~nm}$ \\
\hline $1_{\text {SHIFT }}$ & 12.5 & 0.35 & 10 & $1(10.4)$ & $1(11.8)$ & $1(11.8)$ & 1 \\
\hline $2_{\text {SHIFT }}$ & 12.5 & 0.52 & 10 & 2 & 2 & 2 & 2 \\
\hline $3_{\text {SHIFT }}$ & 20.0 & 0.51 & 10 & $1(13.4)$ & $1(14.1)$ & $1(14.2)$ & 1 \\
\hline $4_{\text {SHIFT }}$ & 20.0 & 0.60 & 10 & $1(12.3)$ & $1(13.0)$ & $1(13.4)$ & 1 \\
\hline $5_{\text {SHIFT }}$ & 20.0 & 0.81 & 10 & 2 & 2 & 2 & $>2$ \\
\hline $1_{\text {CENT }}$ & 20.0 & 0.65 & 20 & Leakage & $1(15.3)$ & $1(15.8)$ & 2 \\
\hline $2_{\text {CENT }}$ & 22.0 & 0.65 & 20 & Leakage & $1(13.9)$ & $1(14.9)$ & 2 \\
\hline $3_{\text {CENT }}$ & 22.0 & 0.67 & 20 & $1(12.6)$ & $1(13.7)$ & $1(14.0)$ & 2 \\
\hline
\end{tabular}

Table 7. Modal properties of the MOFs with the shifted and the central cores.

In theory, the core expansion leads to the increase of normalized frequency $\mathrm{V}$, representing exactly the amount of guided modes. This situation can be simply illustrated with the expression (Birks et al., 1997):

$$
\mathrm{V}=2 \Pi \Lambda \sqrt{\mathrm{n}_{\mathrm{co}}^{2}-\mathrm{n}_{\mathrm{cl}}^{2}} / \lambda
$$

where $\mathrm{n}_{\mathrm{co}}$ and $\mathrm{n}_{\mathrm{cl}}$ are the refractive index of the core and the effective refractive index of the cladding respectively.

So when V-parameter increases, the existing modes become less sensitive to perturbations, for example, to macro- and microbending, and the higher order mode attenuation coefficient has to be lower. The alternative process in our experiment may be explained by the mechanism of the enhanced modal leakage through the spaces between the air holes, which dimensions extend in the direct proportion to $\Lambda$-growth when the whole structure is scaled. Generally, the modal consistence of the MOFs with shifted and central cores approximately coincides if deal with the same core diameters and k-parameters values. The most impressive difference is that in the visible part of the spectra $(\lambda=658 \mathrm{~nm})$ the fundamental mode radiation of the centralcore MOFs intensively leaks away from the core area into the outer fiber cladding, while it good confines in the shifted-core ones. Such high bending resistance properties can be elucidated by the increased air-filling fraction (sample $4_{\mathrm{SHIFT}}$ ) and the mode spot size that is decreased as compared to the spot size in the central-core MOF (samples $4_{\text {SHIFT }}$ and $1_{\text {CENT). In }}$ addition, the mode spot size in MOFs with the shifted core is weakly dependent on the wavelength that is a distinctive feature of MOFs as a class of lightguides with the triangular arrangement of the air holes (Nielsen et al., 2004a). Despite the slight reduction in the fundamental mode spot size, the shifted-core MOFs have an obvious preference over the central-core analog. It consists in the expansion of the spectral operation range. 
Thus, we have accurately determined that the position of bend-induced leakage boundary of the MOFs with the shifted core is located in the visible part of the spectra. To identify it numerically we have produced several samples of the length from 50 to 100 meters. The respective curve is presented in Figure 19.

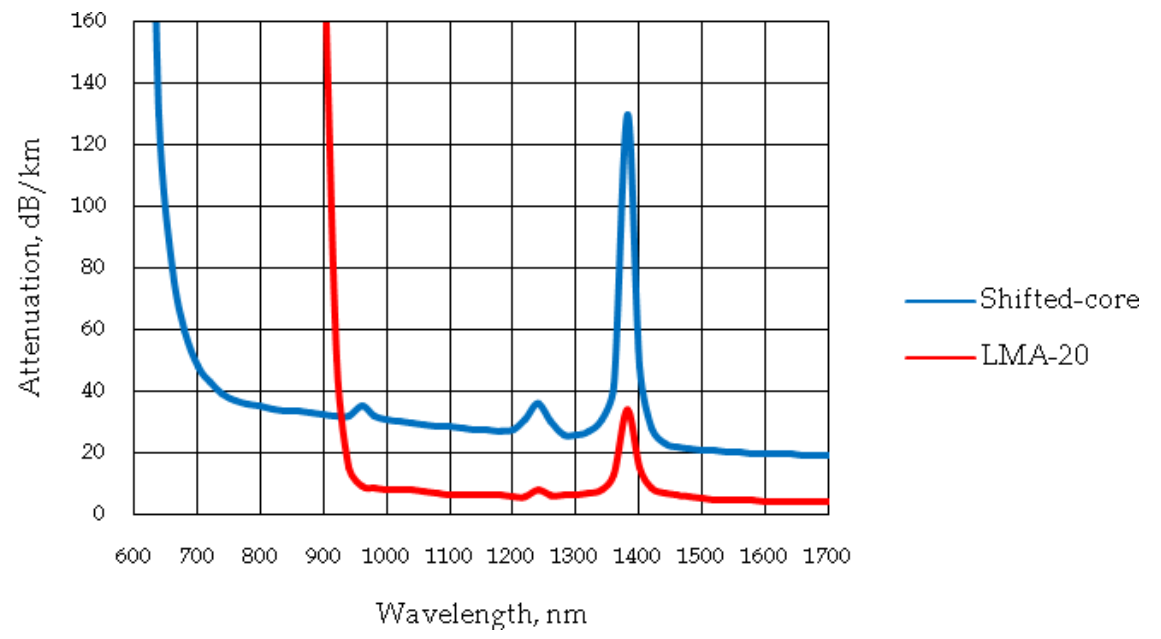

Fig. 19. Spectral attenuation pattern of the MOFs with the core diameter of $20 \mu \mathrm{m}$.

As Figure 19 shows, leakage of the fundamental mode power occurs in the wavelength region $\lambda \sim 650 \mathrm{~nm}$. At the same time, in the central-core MOF with the identical core size strong leakage occurs at $\lambda \sim 900 \mathrm{~nm}$ (spool diameter of 16 centimeters) and at $\lambda \sim 650 \mathrm{~nm}$ (spool diameter of 32 centimeters) (Nielsen et al., 2004b). In other words, the MOF with the shifted core can be characterized by the noticeably improved stationary bending resistance due to the relatively large value of k-parameter which is equal to 0.60 . The spectral operation range widening is about $250 \mathrm{~nm}$ that is an obvious preference over the typical MOF structure.

Similar processes can be observed in the MOF with the shifted core of $34 \mu \mathrm{m}$ in diameter (Figure 20).

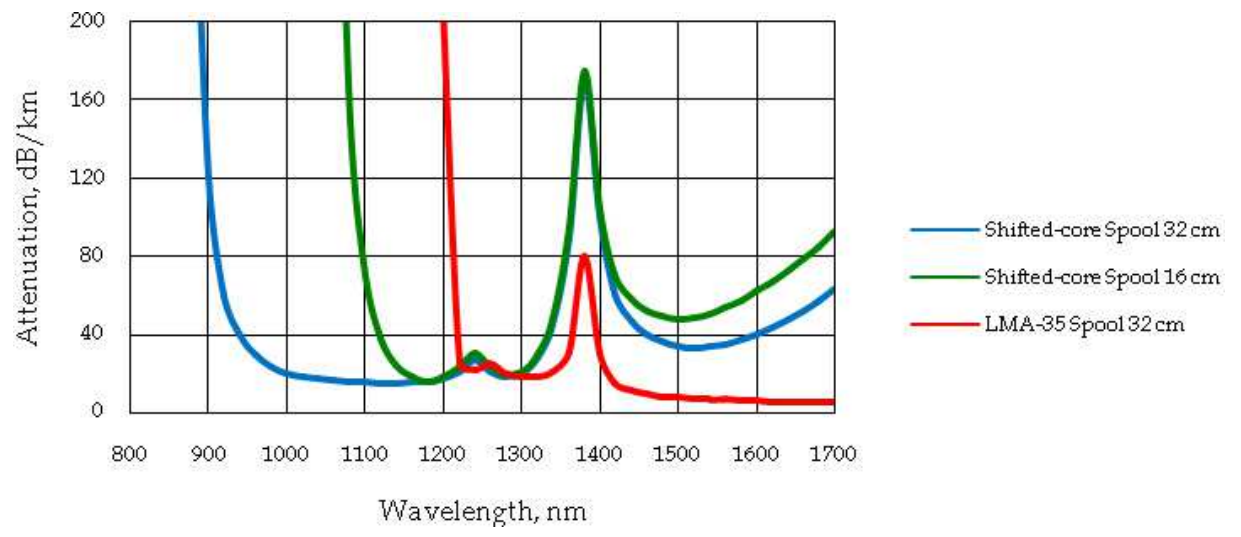

Fig. 20. Spectral attenuation pattern of the MOFs with the core diameter of $34-35 \mu \mathrm{m}$. 
The increase in the k-parameter value up to 0.65 allows the fiber being placed on a standard spool of 16 centimeters in diameter to operate still in the single-mode regime. The mode spot size is approximately $26 \mu \mathrm{m}$ that coincides with the data for the central-core MOFs (Nielsen et al., 2003).

We assert that further increase in the air-filling fraction (up to 0.70), caused by the natural desire to expand the range of working frequencies as much as possible, leads to the display of the impurity of the higher order mode in the power distribution of light at the output end of the fiber. So we consider the MOF with the shifted core of $34-35 \mu \mathrm{m}$ in diameter and the k-parameter of 0.65 to be the optimal bend-resistant design combining robust fundamental mode propagation and the expanded spectral operation interval.

It should be noted that the investigated MOF with the shifted core of $34 \mu \mathrm{m}$ in diameter exhibits a certain growth of the attenuation coefficient in the spectral region $\lambda>1200 \mathrm{~nm}$ (Figure 20). As we have already ascertained mode spot size is weakly dependent on the working wavelength in the fibers with the air holes assembled in the triangular array (Nielsen et al., 2004a) and the fundamental mode leakage occurs in the short-wavelength part of the spectra (Nielsen et al., 2004b). When the fiber core is shifted, the effective mode area largely penetrates into the outer fiber cladding as compared to the central-core fiber, that corresponds adequately with the certain increase in the attenuation coefficient while going to longer wavelengths.

The validity of the ultimate role of differential modal attenuation in achievement of the single-mode operation may be illustrated by the following data (Table 8).

\begin{tabular}{|c|c|c|c|c|}
\hline \multirow{2}{*}{ Length, $\mathrm{m}$} & \multicolumn{4}{|c|}{ Wavelength, $\mathrm{nm}$} \\
\cline { 2 - 3 } & $\lambda=658 \mathrm{~nm}$ & $\lambda=808 \mathrm{~nm}$ & $\lambda=980 \mathrm{~nm}$ & $\lambda=1550 \mathrm{~nm}$ \\
\hline \multicolumn{5}{|c|}{ MOFs with the shifted core and $\mathrm{k}=0.65$} \\
\hline 50 & 1 mode & 1 mode & \multirow{2}{*}{2 modes } \\
\hline 5 & 1 mode & 1 mode & \\
\hline 3 & 1 mode & 2 modes & \\
\hline 1.5 & 2 modes & 2 modes & \multirow{2}{*}{2 modes } \\
\hline \multicolumn{5}{|c|}{ MOFs with the shifted core and $\mathrm{k}=0.68$} \\
\hline 40 & 1 mode & 1 mode & \\
\hline 5 & 1 mode & 2 mode & \\
\hline 2.5 & 2 mode & 2 mode & \\
\hline
\end{tabular}

Table 8. Dependence of the modal consistence on the fiber length.

Table 8 illustrates the modal consistence of the short-length (from 1.5 to 5 meters) samples of the MOFs with the shifted core of $20 \mu \mathrm{m}$ in diameter. We have compared these experimental data with those obtained for the long-length samples. During the measurement procedures the fibers were placed on standard spools of 16 centimeters in diameter. As one can see, short-length samples were characterized by the multimode regime of operation which transformed into the single-mode while increasing the fiber length. The higher order mode attenuated almost completely after passing the length of approximately 5 meters (sample with $\mathrm{k}=0.65)$. On the basis of the statement that total attenuation of the higher order mode could be estimated to be of at least $10 \mathrm{~dB}$, we got the bottom boundary for the attenuation coefficient equal to $\sim 2 \mathrm{~dB} / \mathrm{m}$ at $\lambda=658-808 \mathrm{~nm}$. That result corresponded with the data we have obtained earlier while working on the MOFs with a multi-element core. Table 8 shows 
that further increase in the k-parameter up to 0.68 decreased the attenuation coefficient of the higher order mode that, in turn, limited steadily the spectral operation range.

Finally, we declare that in optimized conditions the expansion of the spectral operation range of the shifted-core MOFs may achieve $300 \mathrm{~nm}$ in the near infrared part of the spectra (Figure 20) as compared to the central-core MOFs (Nielsen et al., 2004b). This effectively combines with the large air-filling fraction $(\mathrm{k}=0.65)$ that makes the fiber less susceptible to macrobending or microbending effects.

\section{Conclusion}

In this work, we have reported on the recent results in investigating main optical properties of several series of MOFs. Two of them (7- and 19-element-core designs) have already been discussed earlier, whereas three others (the circular cladding distribution, the $\mathrm{C}_{3 \mathrm{~V}}$ cladding symmetry and the shifted-core structure) represent novel design. The modal consistence of radiation propagated along the fiber cores and the spectral attenuation curves have been provided.

We have accurately determined that the basic priorities of novel MOF structures are the expanded spectral operation range (up to $300 \mathrm{~nm}$ ) and the bend-resistant performance. We have managed to achieve these features by ensuring proper conditions for the high airfilling fraction of the cladding structure. Simultaneously, we have successfully assured the condition for the higher order mode to undergo strong attenuation via considerable power leakage into the outer fiber cladding. That point principally diverges our approach from the classical one, which guarantees the phase cut-off condition for the propagation of the second order mode. The mechanism of the differential modal attenuation has been clearly stated in the case of the shifted-core MOF design when the core area was located close to the outer fiber cladding. The mentioned circumstance has led to the strong attenuation coefficient of the higher order mode that has been estimated to be in the vicinity of $2-6 \mathrm{~dB} / \mathrm{km}$. In other cases the physical foundation of the differential modal attenuation was not so clear, though it effectively worked allowing the MOFs to support practically only a single transverse mode over a broad spectral range.

The fiber designs discussed in this work can be successfully applied in high power laser technology or in laser beam delivery applications with standardized curve parameters.

\section{References}

Agruzov, P. M.; Kozlov, A. S.; Petrov, M. P.; Dukel'skii, K. V.; Komarov, A. V.; TerNersesyants, E. V.; Khokhlov, A. V. \& Shevandin, V. S. (2008). Mode composition of holey fibers with a large seven-element core. Journal of Optical Technology, Vol. 75, No. 11, (November 2008), pp. 747-749, ISSN 1070-9762

Agruzov, P. M.; Dukel'skii, K. V.; Komarov, A. V.; Ter-Nersesyants, E. V.; Khokhlov, A. V. \& Shevandin, V. S. (2010). Developing microstructured lightguides with a large core, and an investigation of their optical properties. Journal of Optical Technology, Vol. 77, No. 1, (January 2010), pp. 59-62, ISSN 1070-9762

Birks, T. A.; Knight, J. C. \& Russell, P. St. J. (1997). Endlessly single-mode photonic crystal fiber. Optics Letters, Vol. 22, No. 13, (July 1997), pp. 961-963, ISSN 0146-9592 
Dukel'skii, K. V.; Komarov, A. V.; Ter-Nersesyants, E. V.; Khokhlov, A. V. \& Shevandin, V. S. (2005). Realization of photonic crystal fibers in S.I. Vavilov Federal Optical Institute. Proceedings of AIS'05 and CAD'2005, ISBN 5-9221-0621-X, Divnomorsk, September 2005

Dukel'skii, K. V.; Kondrat'ev, Yu. N.; Komarov, A. V.; Ter-Nersesyants, E. V.; Khokhlov, A. V. \& Shevandin, V. S. (2006). How the pitch of a holey optical fiber affects its lightguide properties. Journal of Optical Technology, Vol. 73, No. 11, (November 2006), pp. 808-811, ISSN 1070-9762

Jeunhomme, L. B. (1983). Single-Mode Fiber Optics: Principles and applications. Marcel Dekker, ISBN 978-082-4770-20-4, New York, USA

Knight, J. C.; Birks, T. A.; Russell, P. St. J. \& Atkin, D. M. (1996). All-silica single-mode optical fiber with photonic crystal cladding. Optics Letters, Vol. 21, No. 19, (October 1996), pp. 1547-1549, ISSN 0146-9592

Knight, J. C.; Birks, T. A.; Russell, P. St. J. \& Atkin, D. M. (1997). All-silica single-mode optical fiber with photonic crystal cladding: errata. Optics Letters, Vol. 22, No. 7, (April 1997), pp. 484-485, ISSN 0146-9592

Knight, J. C. (2003). Photonic crystal fibres. Nature, Vol. 424, No. 6950, (August 2003), pp. 847-851, ISSN 0028-0836

Limpert, J.; Deguil-Robin, N.; Manek-Honninger, I.; Salin, F.; Roser, F.; Liem, A.; Schreiber, T.; Nolte, S.; Zellmer, H.; Tunnermann, A.; Broeng, J.; Petersson, A. \& Jakobsen, C. (2005). High-power rod-type photonic crystal fiber laser. Optics Express, Vol. 13, No. 4, (February 2005), pp. 1055-1058, ISSN 1094-4087

Limpert, J.; Schmidt, O.; Rothhardt, J.; Roser, F.; Schreiber, T.; Tunnermann, A.; Ermeneux, S.; Yvernault, P. \& Salin, F. (2006). Extended single-mode photonic crystal fiber lasers, Optics Express, Vol. 14, No. 7, (April 2006), pp. 2715-2720, ISSN 1094-4087

Martelli C.; Canning J.; Gibson B. \& Huntington S. Optics Express, Vol. 15, No. 26, (December 2007), pp. 17639-17644, ISSN 1094-4087

Mortensen, N. A. (2002). Effective area of photonic crystal fibers. Optics Express, Vol. 10, No. 7, (April 2002), pp. 341-348, ISSN 1094-4087

Mortensen, N. A.; Folkenberg, J. R., Skovgaard, P. M. W. \& Broeng, J. (2002). Numerical aperture of single-mode photonic crystal fibers. IEEE Photonic Technology Letters, Vol. 14, No. 8, (August 2002), pp. 1094-1096, ISSN 1041-1135

Mortensen, N. A.; Folkenberg, J. R.; Nielsen, M. D. \& Hansen, K. P. (2003). Modal cut-off and the V-parameter in photonic crystal fibers. Optics letters, Vol. 28, No. 20, (October 2003), pp. 1879-1881, ISSN 0146-9592

Nielsen, M. D.; Folkenberg, J. R. \& Mortensen, N. A. (2003). Single-mode photonic crystal fiber with an effective area of $600 \mu \mathrm{m}^{2}$ and low bending losses. Electronics Letters, Vol. 39, No. 25, (December 2003), pp. 1802-1803, ISSN 0013-5194

Nielsen, M. D.; Folkenberg, J. R.; Mortensen, N. A. \& Bjarklev, A. (2004). Bandwidth comparison of photonic crystal fibers and conventional single-mode fibers. Optics Express, Vol. 12, No. 3, (February 2004), pp. 430-435, ISSN 1094-4087

Nielsen, M. D.; Mortensen, N. A.; Albertsen, M.; Folkenberg, J.R.; Bjarklev, A. \& Bonacinni, D. (2004). Predicting macrobending loss for large-mode area photonic crystal fibers. Optics Express, Vol. 12, No. 8, (April 2004), pp. 1775-1779, ISNN 1094-4087 
Russell, P. St. J. (2006). Photonic-Crystal Fibers. Journal of Lightwave Technology, Vol. 24, No. 12, (December 2006), pp. 4729-4749, ISSN 0733-8724

Saitoh, K.; Tsuchida, Y.; Koshiba, M. \& Mortensen, N. A. (2005). Endlessly single-mode holey fibers: the influence of core design. Optics Express, Vol. 13, No. 26, (December 2005), pp. 10833-10839, ISSN 1094-4087

Snyder, A. W. \& Love, J. D. (1983). Optical Waveguide Theory. Chapman and Hall Ltd, ISBN 978-041-2099-50-2, London, UK

Tsuchida, Y.; Saitoh, K. \& Koshiba, M. (2005). Design and characterization of single-mode holey fibers with low bending losses. Optics Express, Vol. 13, No. 12, (June 2005), pp. 4770-4779, ISSN 1094-4087

Tunnermann, A.; Schreiber, T.; Roser, F.; Liem, A.; Hofer, S.; Zellmer, H.; Nolte, S. \& Limpert, J. (2005). The renaissance and bright future of fibre lasers. Journal of Physics B: Atomic, Molecular and Optical Physics, Vol. 38, No. 9, (April 2005), pp. S681-S693, ISSN 0953-4075 


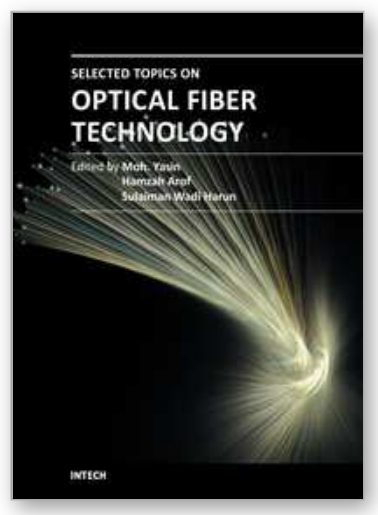

\author{
Selected Topics on Optical Fiber Technology \\ Edited by Dr Moh. Yasin
}

ISBN 978-953-51-0091-1

Hard cover, 668 pages

Publisher InTech

Published online 22, February, 2012

Published in print edition February, 2012

This book presents a comprehensive account of the recent advances and research in optical fiber technology. It covers a broad spectrum of topics in special areas of optical fiber technology. The book highlights the development of fiber lasers, optical fiber applications in medical, imaging, spectroscopy and measurement, new optical fibers and sensors. This is an essential reference for researchers working in optical fiber researches and for industrial users who need to be aware of current developments in fiber lasers, sensors and other optical fiber applications.

\title{
How to reference
}

In order to correctly reference this scholarly work, feel free to copy and paste the following:

Vladimir Demidov, Konstantin Dukel'skii and Victor Shevandin (2012). Design and Characterization of SingleMode Microstructured Fibers with Improved Bend Performance, Selected Topics on Optical Fiber Technology, Dr Moh. Yasin (Ed.), ISBN: 978-953-51-0091-1, InTech, Available from:

http://www.intechopen.com/books/selected-topics-on-optical-fiber-technology/design-and-characterization-ofsingle-mode-microstructured-fibers-with-improved-bend-performance

\section{INTECH}

open science | open minds

\section{InTech Europe}

University Campus STeP Ri

Slavka Krautzeka 83/A

51000 Rijeka, Croatia

Phone: +385 (51) 770447

Fax: +385 (51) 686166

www.intechopen.com

\section{InTech China}

Unit 405, Office Block, Hotel Equatorial Shanghai

No.65, Yan An Road (West), Shanghai, 200040, China

中国上海市延安西路65号上海国际贵都大饭店办公楼405单元

Phone: +86-21-62489820

Fax: +86-21-62489821 
(C) 2012 The Author(s). Licensee IntechOpen. This is an open access article distributed under the terms of the Creative Commons Attribution 3.0 License, which permits unrestricted use, distribution, and reproduction in any medium, provided the original work is properly cited. 